Rev. Int. Contam. Ambie. 35 (4) 957-963, 2019

DOI: 10.20937/RICA.2019.35.04.14

\title{
EVALUACIÓN DE LAS CARACTERÍSTICAS DE CALIDAD Y BROMATOLÓGICAS DE ENSILADOS ELABORADOS CON RESIDUOS DE CALABAZA (Cucurbita argyrosperma)
}

Evaluation of the quality and bromatological characteristics of ensilages made with pumpkin residues (Cucurbita argyrosperma)
Ricardo LORENZO-HERNÁNDEZ ${ }^{1}$, Nicolás TORRES-SALADO ${ }^{1}$, Paulino SÁNCHEZ-SANTILLÁN ${ }^{1 *}$, Jerónimo HERRERA-PÉREZ ${ }^{1}$, Félix de Jesús MAYRÉN-MENDOZA ${ }^{1}$, Teódulo SALINAS-RÍOS ${ }^{2}$, Adelaido Rafael ROJAS-GARCÍA ${ }^{1}$ y María de los Ángeles MALDONADO-PERALTA ${ }^{1}$

${ }^{1}$ Facultad de Medicina Veterinaria y Zootecnia núm 2, Universidad Autónoma de Guerrero, km 197 carretera federal Acapulco-Pinotepa Nacional, 41940 Cuajinicuilapa, Guerrero, México

${ }^{2}$ Facultad de Medicina Veterinaria y Zootecnia, Universidad Autónoma Benito Juárez de Oaxaca, Av. Universidad s/n, Ex Hacienda 5 Señores, 68120 Oaxaca de Juárez, Oaxaca, México

*Autor para correspondencia: paulino_san@hotmail.com

(Recibido: marzo 2018; aceptado: enero 2019)

Palabras clave: pangola, melaza, fermentación, cáscara, pulpa

\section{RESUMEN}

La cáscara con pulpa de calabaza (CPC) es un subproducto agrícola con potencial contaminante que puede usarse para ensilar. El objetivo fue determinar las características de calidad y bromatológicas de ensilados elaborados con CPC, heno de pasto pangola, urea y dos porcentajes de melaza fermentados durante 14 y 21 días. Los tipos de ensilado ( $2 \mathrm{~kg}$ ) fueron los siguientes: E1: $72.5 \%$ de CPC, $22.5 \%$ de pasto, $3 \%$ de melaza y $2 \%$ de urea; E2: $72.5 \%$ de CPC, $19.5 \%$ de pasto, $6 \%$ de melaza y $2 \%$ de urea. Ambos ensilados se fermentaron por 14 y 21 días. Se evaluaron los siguientes elementos: $\mathrm{pH}$, contenido de materia seca (MS), ácido láctico, cenizas (Ce), materia orgánica (MO), proteína cruda (PC), fibra detergente neutro (FDN), fibra detergente ácida (FDA) y hemicelulosa. El análisis estadístico fue un diseño completamente al azar con arreglo factorial $2 \times 2$, con tipos de ensilado y tiempo de fermentación como factores. El pH de E2 no varió (p>0.05) a los 14 y 21 días de fermentación, promediando 3.85. Se cuantificó mayor producción de ácido láctico en E1 a 14 días; asimismo, este ensilado presentó mayor MS que E2 ( $\mathrm{p} \leq 0.05)$. El contenido de PC, FDN, FDA, Ce y hemicelulosa no mostraron diferencias $(p>0.05)$ por tipo de ensilado ni tiempos de fermentación. En conclusión, la CPC puede ensilarse con heno de pasto pangola, urea y hasta $6 \%$ de melaza de caña de azúcar fermentada por 14 o 21 días sin afectar sus características de calidad y bromatológicas.

Key words: pangola, molasses, fermentation, shell, pulp 
were E1 (72.5\% PPS, $22.5 \%$ grass, 3\% molasses and 2\% urea) and E2 (72.5\% PPS, $19.5 \%$ grass, $6 \%$ molasses and $2 \%$ urea). Both ensilages were fermented for 14 and 21 days. The $\mathrm{pH}$ was evaluated, along with dry matter (DM), lactic acid, ash (As), organic matter $(\mathrm{OM})$, crude protein $(\mathrm{CP})$, neutral detergent fiber (NDF), acid detergent fiber (ADF) and hemicellulose content. The statistical analysis was a completely randomized $2 \times 2$ design with ensilage types and fermentation time as factors. The $\mathrm{pH}$ of $\mathrm{E} 2 \mathrm{did}$ not vary $(\mathrm{p}>0.05)$ at 14 and 21 days of fermentation, averaging 3.85. A higher production of lactic acid was quantified with E1 at 14 days, and it also presented a higher DM than E2 ( $\mathrm{p} \leq 0.05)$. The content of CP, NDF, ADF, As and hemicellulose did not show differences $(p>0.05)$ by ensilage type or fermentation times. It is concluded that PPS can be ensiled with pangola grass hay, urea and up to $6 \%$ sugarcane molasses fermented for 14 or 21 days without affecting its quality and bromatological characteristics.

\section{INTRODUCCIÓN}

La calabaza (Cucurbita argyrosperma) es un vegetal nativo de América (Martínez-Valdiviezo et al. 2015) que produce flores, tallos jóvenes, frutos tiernos, frutos maduros y semillas para consumo humano (FAO 2016, Almaguer et al. 2017). En México, los estados de Campeche, Guerrero, Michoacán y Tamaulipas producen calabaza para obtener la semilla, por lo que la cáscara con pulpa se convierte en un subproducto agrícola (Ayvar et al. 2004) que crea un problema ambiental cuando no se usa como alimento no convencional para el ganado y aves de corral (Razzaghzadeh et al. 2007, FAO 2016). La cáscara con pulpa de la calabaza contiene $6.45 \%$ de materia seca (MS), $13.69 \%$ de cenizas, $8.63 \%$ de proteína cruda (PC), $49.39 \%$ de fibra detergente neutra (FDN), $40.45 \%$ de fibra detergente ácida (FDA) y $3.29 \mathrm{Mcal} / \mathrm{kg}$ de energía metabolizable; además, tiene un rendimiento aproximado de $1 \mathrm{t} / \mathrm{ha}$ (Dorantes-Jiménez et al. 2016)

El proceso de ensilaje permite la fermentación de carbohidratos solubles a ácido láctico para conservar los nutrientes del material ensilado (Bezabih y Tamir 2014, Garcés et al. 2014) por la disminución del pH e inhibición de microorganismos inductores de la putrefacción (Garcés et al. 2014). Además, se usa urea agrícola como fuente de nitrógeno no proteico (Garcés et al. 2014) para mejorar el contenido de nitrógeno de los ensilados y la melaza de caña de azúcar como fuente de azucares solubles (Guzmán et al. 2012, Garcés et al. 2014) para mejorar la fermentación por las bacterias ácido lácticas (AraizaRosales et al. 2013).

El uso de subproductos agrícolas en la alimentación animal como ensilados es una alternativa viable en el trópico, ya que permite aumentar la calidad del producto final, reduce costos de producción y se aprovechan los residuos agrícolas potencialmente contaminantes (Guzmán et al. 2012). Por tanto, surge la pregunta de si los azúcares contenidos en la cáscara con pulpa de calabaza y la melaza permitirían mejorar o conservar las características de calidad y bromatológicas de los ensilados, porque se usa heno de pasto pangola altamente lignificado para contrarrestar la humedad en dicha cáscara con pulpa en tiempos cortos de fermentación.

En este sentido, la inclusión de 3 y $6 \%$ de melaza en la elaboración de ensilados de cáscara con pulpa de calabaza con heno de pasto pangola permite tiempos cortos de fermentación sin afectar las características de calidad y bromatológicas de los ensilados, que pueden ser una alternativa para la alimentación animal en regiones tropicales de México. Por tanto, el objetivo de este trabajop fue determinar las características de calidad y bromatológicas de ensilados elaborados a partir de cáscara con pulpa de calabaza, heno de pasto pangola, urea y dos porcentajes de melaza de caña de azúcar fermentados durante 14 y 21 días.

\section{MATERIALES Y MÉTODOS}

El presente trabajo se llevó a cabo en el Laboratorio de Nutrición Animal de la Facultad de Medicina Veterinaria y Zootecnia núm. 2 de la Universidad Autónoma de Guerreo, ubicado en el kilómetro 197 de la carretera Acapulco-Pinotepa Nacional en Cuajinicuilapa, Guerrero, México. El municipio de Cuajinicuilapa se ubica en la región de la Costa Chica del Estado de Guerrero; está situado a $16^{\circ} 08^{\prime}$ de latitud norte y $98^{\circ} 23^{\prime}$ de longitud oeste, a una altura de $50 \mathrm{msnm}$ (GEG 2017).

\section{Ensilados}

Los ingredientes con base húmeda para elaborar los ensilados fueron cáscara con pulpa de calabaza 
(Cucurbita argyrosperma), heno de pasto pangola (Digitaria decumbens) cosechado y empacado a los 120 días de rebrote, melaza de caña de azúcar y urea. La composición bromatológica de la cáscara con pulpa de calabaza y el heno de pasto pangola se presenta en el cuadro I. La cáscara con pulpa de calabaza y el pasto pangola se molieron en un molino mixto (M.A.GRO® TR-3500, México) con criba de 2.54 $\mathrm{cm}$ de diámetro. Los ensilados $(2 \mathrm{~kg})$ se elaboraron de la siguiente manera: E1: $72.5 \%$ de cáscara con pulpa de calabaza, $22.5 \%$ de heno de pasto pangola, $3 \%$ de melaza de caña y $2 \%$ de urea; y E2: $72.5 \%$ de cáscara con pulpa de calabaza, $19.5 \%$ de heno de pasto pangola, $6 \%$ de melaza de caña y $2 \%$ de urea.

CUADRO I. ANÁLISIS BROMATOLÓGICO DE LA CÁSCARA CON PULPA DE CALABAZA Y DEL HENO DE PASTO PANGOLA

\begin{tabular}{lcc}
\hline Variable (\%) & $\begin{array}{c}\text { Cáscara con } \\
\text { pulpa de calabaza }\end{array}$ & $\begin{array}{c}\text { Heno de } \\
\text { pasto pangola }\end{array}$ \\
\hline Materia seca & 13.71 & 92.36 \\
Proteína cruda & 15.40 & 6.57 \\
Fibra detergente neutro & 48.01 & 70.75 \\
Fibra detergente ácido & 24.21 & 39.68 \\
Hemicelulosa & 23.80 & 31.07 \\
Cenizas & 13.78 & 6.13 \\
Materia orgánica & 86.22 & 93.87 \\
\hline
\end{tabular}

Los ensilados se elaboraron en bolsas de propileno $(40 \times 40 \mathrm{~cm})$ y el aire contenido se extrajo con una aspiradora (Koblenz ${ }^{\circledR}$, España) para proporcionar las condiciones de anaerobiosis requeridas para iniciar el proceso de fermentación. Las bolsas se cerraron con rafia para mantener las condiciones de anaerobiosis y se fermentaron durante 14 y 21 días en una galera con temperatura ambiente promedio de $33{ }^{\circ} \mathrm{C}$.

\section{Análisis de calidad de los ensilados}

Al finalizar cada tiempo de fermentación se realizaron análisis para determinar la calidad de los ensilados. Para determinar el $\mathrm{pH}$, en un vaso de precipitados de $100 \mathrm{~mL}\left(\operatorname{Kimax}^{\circledR}\right)$ se colocaron $20 \mathrm{~g}$ de ensilado y se agregaron $50 \mathrm{~mL}$ de agua destilada (pH 7). El contenido se agitó cada 15 min durante 3 h; luego se filtró con una gasa doble y se depositó en un vaso de precipitados $(10 \mathrm{~mL})$ para medir el $\mathrm{pH}$ con un potenciómetro (Hanna ${ }^{\circledR}$ HI2211, Italia; calibración pH 7 y 4) (Silva y Queiroz 2002). El contenido de MS (método 930.15) se estimó según lo descrito por la AOAC (2005).
El ácido láctico se determinó con la metodología de Kimberley y Taylor (1996). En un vaso de precipitados $(100 \mathrm{~mL})$ se colocaron $1 \mathrm{~g}$ de un ensilado y $10 \mathrm{~mL}$ de agua destilada. El vaso de precipitados se mantuvo en refrigeración a $4{ }^{\circ} \mathrm{C}$ por $20 \mathrm{~min}$; posteriormente, se incubó a $22{ }^{\circ} \mathrm{C}$ durante $20 \mathrm{~min}$, con intervalos de agitación de $10 \mathrm{~min}$. El contenido del vaso de precipitados se filtró con una gasa doble y $1 \mathrm{~mL}$ del filtrado se colocó en un tubo Eppendorf de $2 \mathrm{~mL}$ (Neptune ${ }^{\circledR}$, México) con $0.25 \mathrm{~mL}$ de ácido metafosfórico al $25 \%$ (Meyer $\left.^{\circledR}\right)$. La muestra se diluyó $(1: 50)$ con agua destilada; $0.5 \mathrm{~mL}$ se mezclaron con $3 \mathrm{~mL}$ de $\mathrm{H}_{2} \mathrm{SO}_{4}\left(\right.$ Baker $\left.^{\circledR}, 97.3 \%\right)$ en un tubo de ensayo $\left(\operatorname{Kimax}^{\circledR} ; 18 \times 150 \mathrm{~mm}\right)$ y se incubaron a $100{ }^{\circ} \mathrm{C}$ por 10 min en una estufa (Riossa ${ }^{\circledR} \mathrm{HCF}-41$, México). Cuando la temperatura se redujo a $25^{\circ} \mathrm{C}$, se adicionaron $50 \mu 1$ de $\mathrm{CuSO}_{4} .5 \mathrm{H}_{2} \mathrm{O}\left(\mathrm{Merk}^{\circledR} ; 4 \%\right)$ y $100 \mu 1$ de p-hidroxidifenil (Sigma-Aldrich ${ }^{\circledR} ; 1.5$ $\%$ ) e inmediatamente se mezclaron en un vórtex (Genie $2^{\circledR}$, Daigger, USA). Las muestras reposaron 30 min y se midió la absorbancia a $570 \mathrm{~nm}$ en un espectrofotómetro UV-Vis (Jenway ${ }^{\circledR} 6850 \mathrm{UV}$, Reino Unido). Para la curva estándar se preparó una solución de lactato de litio $0.1 \%(538.6 \mathrm{mg}$ de lactato de litio [Sigma-Aldrich ${ }^{\circledR}$ ] en $500 \mathrm{~mL}$ de agua destilada).

\section{Análisis bromatológico}

Las muestras de ensilados se deshidrataron en una estufa (Riossa ${ }^{\circledR} \mathrm{HCF}-41$, México) a $60{ }^{\circ} \mathrm{C}$ por $72 \mathrm{~h}$. Posteriormente, se molieron usando una criba de $1 \mathrm{~mm}$ de diámetro en un molino Thomas-Wiley Mill (Thomas Scientific ${ }^{\circledR}$, Swedesboro, NJ, EUA). En las muestras se determinaron el contenido de PC (método 920.105) y Ce (método 942.05) con los procedimientos descritos por la AOAC (2005). La FDN y la FDA se determinaron con el método descrito por van Soest et al. (1991). La hemicelulosa se calculó por diferencia entre FDN y FDA.

\section{Análisis estadístico}

Las variables de calidad de los ensilados y carcaterísticas bromatológicas (cuatro repeticiones independientes por interacción) se analizaron en un diseño completamente al azar con arreglo factorial $2 \times 2$. Los factores fueron el tipo de ensilado (E1 y E2) y el tiempo de fermentación (14 y 21 días). Los promedios se ajustaron por mínimos cuadrados para compararlos con la prueba de Tukey $(\alpha=0.05)$. El modelo estadístico fue el siguiente:

$Y_{\mathrm{ijk}}=\mu+A_{\mathrm{i}}+B_{\mathrm{j}}+(A B)_{\mathrm{ij}}+\varepsilon_{\mathrm{ijk}}$ 
donde $Y_{\mathrm{ijk}}$ es la variable de respuesta, $\mu$ la media general, $A_{\mathrm{i}}$ el efecto del $i$-ésimo factor tipo de ensilado, $B_{\mathrm{j}}$ el efecto del $j$-ésimo factor tiempo de fermentación, $(A B)_{\mathrm{ij}}$ el efecto de la interacción entre tipo de ensilado y tiempo de fermentación, y $\varepsilon_{\mathrm{ijk}} \mathrm{el}$ error aleatorio asociado a la $k$-ésima repetición dentro de la interacción entre tipo de ensilado y tiempo de fermentación.

\section{RESULTADOS Y DISCUSIÓN}

El pH y la concentración de ácido láctico en los ensilados fueron afectados por la interacción entre tipos de ensilado y tiempo de fermentación ( $\mathrm{p} \leq$ 0.05; Cuadro II), mientras que el contenido de MS, PC, FDN, FDA, hemicelulosa, Ce y materia orgánica $(\mathrm{MO})$ no mostraron interacción $(\mathrm{p}>0.05$; Cuadro II). Es importante destacar que los tiempos de fermentación evaluados en el presente estudio se establecieron en los valores señalados porque la calabaza contiene $45 \%$ de carbohidratos (Guenkov 1974) fácilmente fermentables para producir ácido láctico, lo cual reduce el $\mathrm{pH}$, preserva los nutrientes e impide el crecimiento de microorganismos indeseables (Amanullah et al. 2014) en tiempos más cortos de fermentación.

E2 no afectó ( $p>0.05$; Cuadro II) el pH cuando se fermentó por 14 y 21 días, ya que se registraron valores promedio de 3.85 . El pH de E1 a los 21 días de fermentación fue $8.8 \%$ mayor $(\mathrm{p} \leq 0.05)$ que a los 14 días (Cuadro II). Los valores de $\mathrm{pH}$ de los ensilados en el presente estudio son menores que los reportados por Hashemi y Razzaghzadeh (2007) y Halik et al. (2014), y similares a los observados por Lozicki et al. (2015). Estos autores publicaron valores de 4.5 en ensilados elaborados con $80 \%$ de calabaza y $20 \%$ de remolacha seca (Halik et al. 2014); 4.85 en ensilados elaborados con $71.4 \%$ de residuos de calabaza, $28.6 \%$ de heno de trigo y $20 \%$ de melaza (Hashemi y Razzaghzadeh 2007); y 3.96 en ensilados de calabaza con pulpa seca de remolacha azucarera (Lozicki et al. 2015).

El ácido láctico es eficaz para reducir el $\mathrm{pH}$ del ensilaje, conservando la calidad del forraje (Meneses et al. 2007). E1 presentó mayor producción de ácido láctico ( $\mathrm{p} \leq 0.05$; Cuadro II) a los 14 días de fermentación; dentro del mismo tipo de ensilado el valor fue $13.3 \%$ mayor que a los 21 días de fermentación, y fue $11.52 \%$ mayor que E2 en ambos tiempos de fermentación evaluados (Cuadro II). Resultados inferiores fueron publicados por Halik et al. (2014) y Lozicki et al. (2015), quienes reportaron 1.7 y $5.6 \%$ de ácido láctico en ensilados elaborados con calabaza, respectivamente.

El contenido de MS de E1 fue $6.61 \%$ superior al de E2 ( $p \leq 0.05$; Cuadro II). Sin embargo, el tiempo de fermentación no afectó el contenido de MS ( $p>0.05$ ), que promedió $32.93 \%$ (Cuadro II). Resultados inferiores fueron reportados por Hashemi y Razzaghzadeh (2007), Halik et al. (2014) y Lozicki et al. (2015), quienes publicaron $30.3 \%$ de MS en ensilados elaborados con $80 \%$ de calabaza y $20 \%$ de remolacha seca (Halik et al. 2014); 30.6\% de MS en ensilados elaborados con $71.4 \%$ de residuos de calabaza, $28.6 \%$ de heno de trigo y $20 \%$ de melaza (Hashemi y Razzaghzadeh 2007), y $29.7 \%$ de MS en ensilados de calabaza con pulpa seca de remolacha azucarera (Lozicki et al. 2015).

La MS, el pH y el ácido láctico se usan para determinar la calidad de un ensilado; por ello, los ensilados que presentan menos de $30 \%$ de MS desarrollan una fermentación dominada por bacterias productoras de ácido butírico y exhiben pérdida de nutrientes digeribles por filtración (Murdoch 1961, Babaeinasab et al. 2015). Por este motivo, en el presente estudio se realizó un ensilado con heno de pangola, ya que la calabaza contiene $86.3 \%$ de humedad. El ácido láctico se produce por la fermentación de sustratos ricos en carbohidratos (Serna y Rodríguez 2005) y la cáscara con pulpa de calabaza contiene $45 \%$ de azucares fácilmente fermentables (Guenkov 1974).

Así, al aumentar la producción de ácido láctico durante el proceso de ensilaje, la concentración de iones de hidrógeno aumentó y el $\mathrm{pH}$ disminuyó a valores inferiores a 5, los cuales inhiben el crecimiento de Clostridum sp. y enterobacterias, no deseados en un proceso de ensilaje (Amanullah et al. 2014, Yitbarek y Tamir 2014). Yitbarek y Tamir (2014) mencionaron que los ensilados que contienen de 25 a $35 \%$ de MS, requieren un $\mathrm{pH}$ de 4.35 a 4.60 (rango superior al reportado en el presente trabajo) para garantizar la estabilidad del ensilado.

La cáscara con pulpa de calabaza mostró $42.6 \%$ más PC que el heno de pasto pangola (Cuadro I). No obstante, el contenido de PC no presentó diferencia $(p>0.05)$ entre tratamientos y tiempos de fermentación (Cuadro II), promediando 26.59 y $26.61 \%$ de PC, respectivamente. Los contenidos de PC de los ensilados en el presente trabajo son superiores a los reportados por Halik et al. (2014) y Lozicki et al. (2015), quienes publicaron niveles de $10.9 \mathrm{y}$ $10.4 \%$, respectivamente, en ensilados elaborados con calabaza y remolacha seca. La diferencia en el contenido de PC se explica porque los trabajos de los autores citados se encuentran dentro del intervalo de 


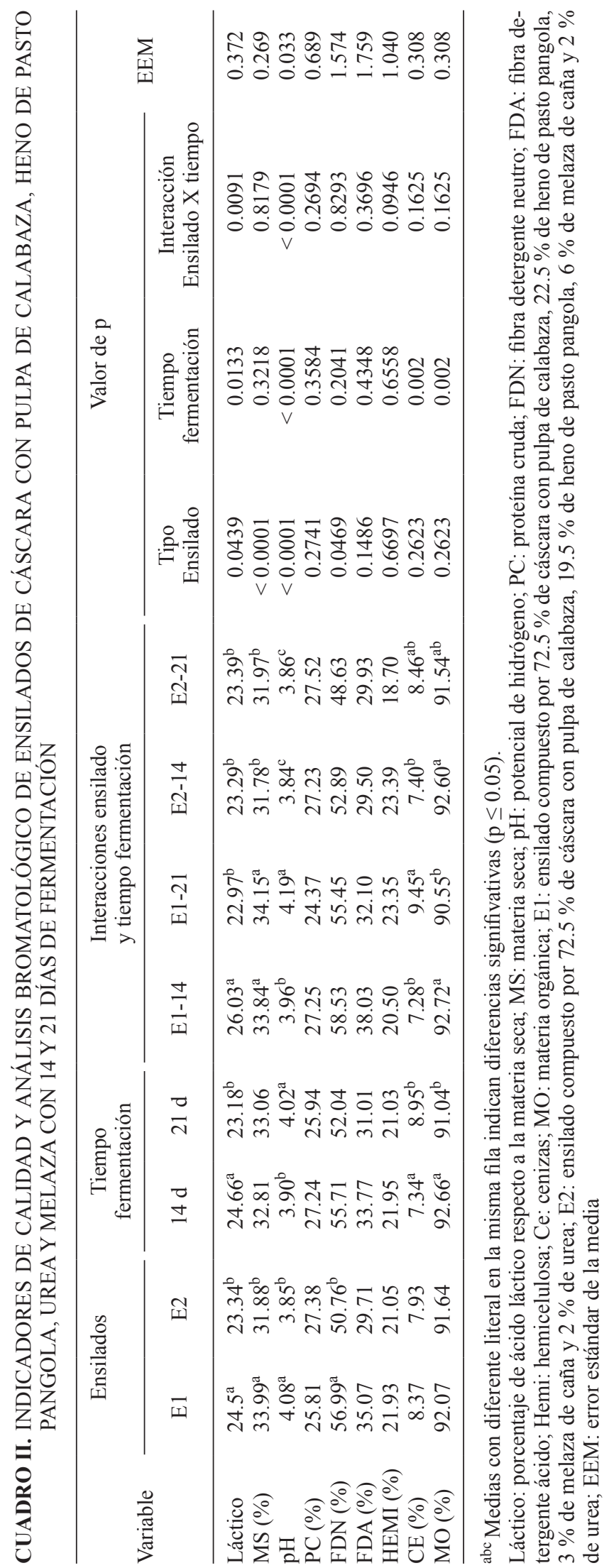


PC en los ensilados ( 8 a $10 \%)$ y valores superiores como los de este trabajo se deben a la adición de urea y probablemente al bajo contenido de almidón (de la Roza-Delgado 2005). Lo anterior se explica porque la calabaza contiene $16.18 \%$ de almidón (Yin y Wang 2016), $55.82 \%$ menos que el grano de maíz (FAO 1993), el cual regularmente se usa en la elaboración de ensilados.

E1 contiene mayor FDN que E2 ( $\mathrm{p} \leq 0.05$; Cuadro II) por la composición inicial de los ensilados, ya que el primero incluye $3 \%$ más heno de pasto pangola que E2, y los pastos maduros alcanzan hasta $70 \%$ de FDN (de la Roza-Delgado 2005). Por su parte, los tiempos de fermentación (Cuadro II) no afectaron el contenido de FDN de los ensilados (p $>0.05$ ). Los tipos de ensilados y los tiempos de fermentación (Cuadro II) no afectaron $(\mathrm{p}>0.05)$ el contenido de FDA, promediando $32.39 \%$ de FDA. Halik et al. (2014) y Lozicki et al. (2015) publicaron valores inferiores de FDN (31.4 y 30.9 $\%$, respectivamente) y FDA (21.3 y $21.6 \%$, respectivamente) en ensilados elaborados con calabaza y remolacha seca.

El contenido de FDN y FDA de los ensilados se relaciona con la proporción de FDN de los ingredientes usados en la elaboración. Halik et al. (2014) y Lozicki et al. (2015) usaron remolacha seca (47.6 $\%$ de FDN y $29.8 \%$ de FDA) para contrarrestar la humedad de la calabaza, la cual contiene $23.15 \%$ menos FDN que el heno de pasto pangola (Lozicki et al. 2015) (Cuadro I), ingrediente usado en el presente estudio para controlar los niveles de humedad en el ensilado propiciados por la cáscara con pulpa de la calabaza.

E1 y E2 no mostraron diferencias $(p>0.05)$ en el contenido de Ce y MO (Cuadro II). Sin embargo, el contenido de Ce a los 21 días de fermentación fue mayor $(\mathrm{p} \leq 0.05)$ que a los 14 días (Cuadro II). En contraste, el contenido de MO en los ensilados fue mayor $(\mathrm{p} \leq 0.05)$ a los 14 que a los 21 días de fermentación (Cuadro II). Estos resultados son inferiores respecto al contenido de Ce y similares con relación a la $\mathrm{MO}$, en ensilados elaborados con calabaza y remolacha seca (Halik et al. 2014, Lozicki et al. (2015).

El contenido de hemicelulosa no mostro diferencias entre tipos de ensilado o por tiempo de fermentación (Cuadro II), con un valor promedio de $21.5 \%$. Esto se debió a que, en el proceso de ensilaje, los azucares fácilmente fermentables son utilizados por los microorganismos para la producción de ácido láctico (Guenkov 1974, Serna y Rodríguez 2005), no así la hemicelulosa.

\section{CONCLUSIONES}

La cáscara con pulpa de calabaza es una alternativa para la elaboración de ensilados con características de calidad y bromatológicas apropiadas, que pueden destinarse a la alimentación de rumiantes. Además, la cáscara con pulpa de calabaza puede ensilarse con heno de pasto pangola, urea y hasta $6 \%$ de melaza de caña de azúcar fermentada por 14 o 21 días sin afectar las características de calidad y bromatológicas de los ensilados.

\section{AGRADECIMIENTOS}

Al Cuerpo Académico UAGro-CA-183 de la Facultad de Medicina Veterinaria y Zootecnia núm. 2 de la Universidad Autónoma de Guerrero, por el financiamiento para la presente investigación "Producción sustentable de rumiantes en el trópico".

\section{REFERENCIAS}

Almaguert J., García H. J., Padilla M. y González M. (2017). Dieta de la milpa modelo de alimentación mesoamericana biocompatible [en línea]. www.gob. $\mathrm{mx} / \mathrm{cms} /$ uploads/attachment/file/98453/La_Dieta_de_ la_Milpa.pdf 13/06/2017

Amanullah S., Kim D., Lee H., Joo Y., Kim S. y Kim C. (2014). Effects of microbial additives on chemical composition and fermentation characteristics of barley silage. Asian Austral. J. Anim. 27 (4), 511-517.

DOI: 10.5713 /ajas.2013.13617

AOAC (2005). Official methods of analysis. 18th ed. Association of Official Analytic Chemists, Washington, D.C., EUA, 1094 pp.

Araiza-Rosales E., Delgado-Licón E., Carrete-Carreón F., Medrano-Roldán H., Solís-Soto A., Murillo-Ortiz M. y Haubi-Segura C. (2013). Degradabilidad ruminal in situ y digestibilidad in vitro de diferentes formulaciones de ensilados de maíz-manzana adicionados con melaza. Av. Investig. Agropecu. 17, 79-96.

Ayvar S., Mena A., Cortés D., Durán J.A. y de Luna J.G. (2004). Rendimiento de la calabaza pipiana en respuesta a la poda y la densidad de población. Rev. Fitotec. Méx. 27 (Esp. 1), 69-72.

Babaeinasab Y., Rouzbehan Y. y Fazaeli H.R.J. (2015). Chemical composition, silage fermentation characteristics, and in vitro ruminal fermentation parameters of potato-wheat straw silage treated with molasses and lactic acid bacteria and corn silage. J. Anim. Sci. 93 (9), 4377-4386. DOI: 10.2527/jas.2015-9082 
Bezabih M. y Tamir B. (2014). Silage additives: Review. Open J. App. Sci. 4, 258-274.

DOI: $10.4236 /$ ojapps.2014.45026

De la Roza-Delgado B. (2005). El ensilado en zonas húmedas y sus indicadores de calidad. Memorias. IV Jornada de Alimentación Animal. Lalín, Pontevedra, España. 7 de octubre. CD-ROM.

Dorantes-Jiménez J., Flota-Bañuelos C., Candelaria-Martínez B., Ramírez-Mella M. y Crosby-Galván M.M. (2016). Calabaza chihua (Curcubita argyrosperma Huber), alternativa para alimentación animal en el trópico. Agroproductividad 9 (9), 33-37.

FAO (1993). Composición química y valor nutritivo del maíz. Organización de las Naciones Unidas para la Alimentación [en línea]. http://www.fao.org/docrep/ t0395s/T0395S00.htm 15/11/2017

FAO (2016). Neglected crops 1492 from a different perspective. Organización de las Naciones Unidas para la Alimentación [en línea]. www.fao.org/docrep/t0646e/ T0646E09.htm\#Cucurbita 20/10/2016

Garcés M., Berrio L., Ruiz S., Serna J. y Builes A. (2014). Ensilaje como fuente de alimentación para el ganado. Rev. Lasallista Mex. 1 (1), 66-71.

GEG (2017) Cuajinicuilapa. Gobierno del Estado de Guerrero [en línea]. http://guerrero.gob.mx/municipios/ costa-chica/cuajinicuilapa/ 11/11/2017

Guenkov G. (1974). Fundamentos de la horticultura cubana. Instituto Cubano del Libro, La Habana, Cuba, $350 \mathrm{pp}$.

Guzmán O., Lemus C., Martínez S., Bonilla J., Plasencia A. y Ly J. (2012). Características químicas del ensilado de residuos de mango (Mangifera indica $\mathrm{L}$.) destinados a la alimentación animal. Rev. Cub. Cienc. Agríc. 46, 369-374.

Halik G., Lozicki A., Koziorzebska A., Dymnicka M. y Arkuszewska E. (2014). Effect of ensiling pumpkin Cucurbita maxima with the addition of inoculants or without it on chemical composition and quality silages. Ann. Warsaw Univ. Life Sci.-Anim. Sci. 53, 103-110.

Hashemi A. y Razzaghzadeh S. (2007). Investigation on the possibility of ensiling cucurbit (Cucurbita pepo) residues and determination of best silage formula. J. Anim. Vet. Adv. 6 (12), 1450-1452.

Kimberley A. y Taylor C. (1996). A simple colorimetric assay for muramic acid and lactic acid. Appl. Biochem. Biotechnol. 56 (1), 49-54.

DOI: $10.1007 / \mathrm{BF} 02787869$
Lozicki A., Koziorzebska A., Halik G., Dymnicka M., Arkuszewska E., Niemiec T. y Bogdan J. (2015). Effect of ensiling pumpkin (Cucurbita maxima D.) with dried sugar beet pulp on the content of bioactive compounds ensilage and its antioxidant potential. Anim. Feed Sci. Technol. 206, 108-113.

DOI: $10.1016 /$ j.anifeedsci.2015.05.012

Martínez-Valdiviezo D., Gómez P., Frand R., AlonsoMoraga A. y del Río-Celestino M. (2015). Physical and chemical characterization in fruit from 22 summer squash (Cucurbita pepo L.) cultivars. Food Sci. Technol. 64 (2), 1225-1233.

DOI: 10.1016/j.lwt.2015.07.023

Meneses M., Megías M., Madrid J., Martínez A., Hernández F. y Oliva J. (2007). Evaluation of the phytosanitary, fermentative and nutritive characteristics of the silage made from crude artichoke (Cyanara scolymus L.) by-product feeding for ruminants. Small Rumin. Res. 70, 292-296. DOI: 10.1016/j.smallrumres.2006.05.008

Murdoch J. (1961). A review of silage-making techniques. Grass Forage Sci. 16 (4), 253-259.

DOI: $10.1111 / j .1365-2494.1961 . t b 00248 . x$

Razzaghzadeh S., Amini-jabalkandi J. y Hashemi A. (2007). Effects of different levels of pumpkin (Cucurbita pepo) residue silage replacement with forage part of ration on male buffalo calves fattening performance. Ital. J. Anim. Sci. 6 (Suppl. 2), 575-577.

DOI: $10.4081 /$ ijas.2007.s2.575

Serna L. y Rodríguez A. (2005). Producción biotecnológica de ácido láctico: estado del arte. Cienc. Tecnol. Aliment. 5 (1), 54-65. DOI: 10.1080/11358120509487672

Silva D.J. y Queiroz A.C. (2002). Análises de alimentos: métodos químicos e biológicos. 3a ed. Viçosa UFV, Brasilia, Brasil, 235 pp.

Van Soest P.J., Roberton J.B. y Lewis B.A. (1991). Methods for dietary fiber, neutral detergent fiber, and nonstarch polysaccharides in relation to animal nutrition. J. Dairy Sci. 74 (10), 3583-3597. DOI: $10.3168 /$ jds.S0022-0302(91)78551-2

Yin L. y Wang C. (2016). Morphological, thermal and physicochemical properties of starches from squash (Cucurbita maxima) and pumpkin (Cucurbita moschata). J. Hortic. 3 (4), 187. DOI: $10.4172 / 2376-0354.1000187$

Yitbarek M. y Tamir B. (2014). Silage additives: Review. Open J. App. Sci. 4, 258-274. DOI: $10.4236 /$ ojapps.2014.45026 